\title{
Advantages of isolated application of fibrin glue over suture in preservation of second-grade spleen lesion
}

\author{
Jovanovic $\mathrm{M}^{1,2}$, Djordjevic $\mathrm{N}^{2}$, Djordjevic $\mathrm{P}^{2}$, Damnjanovic $\mathrm{Z}^{2}$, Smiljkovic $\mathrm{I}^{2}$, Radojkovic $\mathrm{M}^{1,3}$
}

Medical Faculty of Nis, Serbia. milan.m.jovanovic@gmail.com

\begin{abstract}
Objectives: The aim of this procedure was to assess the safety and efficiency of glue application in the treatment of lienal second-degree lesions, compared with traditional suture method of treatment. Background: The spleen is the most frequently injured intra-abdominal structure regardless of injury mechanisms. Fibrin glue is a useful bio-adhesive for safe and efficient treatment of diffuse parenchymatous spleen haemorrhages.

Methods: Seventy dogs under intravenous anaesthesia were subdued to spleen incision in length of $5 \mathrm{~cm}$ and depth of $2 \mathrm{~cm}$. Forty animals from the experimental group were treated with fibrin glue preservation, and 30 animals from the control group were treated with suture. Removed spleens were histologically analyzed. No animal was sacrificed.

Results: The application of fibrin glue allowed reparation to be accomplished in all cases. The effectiveness of primary repair was registered in $95 \%$, with only $5 \%$ of cases requiring an application of fibrin omentoplasty. Secondary bleeding was registered only in $1(2.5 \%)$ case. Preservation with suture is primarily effective only in $40 \%$, requires additional repair in $23 \%$, and even $37 \%$ of cases are not feasible. This method is characterized by mortality of $21 \%$, secondary haemorrhage (32\%), appearance of focal necrosis (42\%) and abscess $(11 \%)$. Conclusions: Application of FG is a method of choice in cases of second-degree transcapsular spleen lesions and it has a considerable advantage over suture (Tab. 4, Fig. 6, Ref. 24). Full Text in PDF www.elis.sk. Key words: spleen injury, spleen preservation, fibrin glue, spleen suture.
\end{abstract}

The fragile structure of spleen parenchyma, thin capsule, plenty of blood in the spleen and its anatomic localisation are the main causes determining the high frequency of spleen injuries as a result of blunt and penetrating types of abdominal trauma (1). The spleen is the most frequently injured intra-abdominal structure regardless of injury mechanisms (2).

Literature data show that splenectomy patients, despite their age and indications for surgical treatment, are very liable to develop fulminant post-splenectomy sepsis, the entity being named "Overwhelming Postsplenectomy Sepsis Syndrome" (OPSS). Many multicentric studies have showed that splenectomy increases the death risk mainly due to developing OPPS by more than 200 times when compared with healthy persons with intact spleen. The global mortality rate of OPPS is between $50-85 \%$ and is significantly higher compared to septic mortality in general population of children $0.01 \%(3-5)$.

A huge multicentric study that involved 2,795 splenectomy patients from 14 surgical centres has showed that $4.25 \%$ of patients developed fulminant sepsis with $59.7 \%$ mortality rate. When comparing the splenectomy patients with general population, the

${ }^{1}$ Medical Faculty of Nis, Serbia, ${ }^{2}$ Vascular Surgery Clinic, Clinical Centre of Nis, Serbia, and ${ }^{3}$ General Surgery Clinic, Clinical Centre of Nis, Serbia

Address for correspodence: M.M. Jovanovic, Clinical Center of Nis, Vascular Surgery Clinic, Bulevar Zorana DinDica 48, 18000 Nis, Serbia. Phone: +381.18 .506971$ incidence of sepsis is 65 -fold higher and the mortality rate is 200 fold higher in the former group of patients. It was showed that the incidence of OPSS in adults was 58 times higher compared to non-splenectomised patients. Iatrogenic splenectomy during long surgical interventions results in high rate of sepsis, which is at least 86 times more frequent in surgically treated adults compared to non-operated patients (6-8). The duration of vulnerability period especially in the development of fulminate pneumonia after splenectomy is unknown. Average time intervals between splenectomy and OPSS appearance vary from several weeks to years and decades. Usually, the risk for life-threatening sepsis is not limited and it can be lifelong $(9,10)$.

Until recently all spleen injuries have been routinely surgically treated by splenectomy despite their grade and clinical presentations. New data especially in the field of immune spleen functions and the role of spleen in body defence against life-threatening systemic infections give grounds for the tendency to lean towards surgical treatment favouring the preservation of injured spleen, and conservative treatment $(10,11)$.

Fibrin glue (FG) is a useful bio-adhesive for safe and efficient treatment of diffuse parenchymatous spleen haemorrhage. In the past few years, it has become important for spleen haemostasis and parenchymatous organ treatment especially in preservative surgical approach to spleen injuries $(12,13)$. In order to estimate the exact chemostatic adhesive ability of FG, the experimental model is designed to simulate spleen lesions with an incision of 
the capsule and parenchyma. Due to large bleeding caused by the section of trabecular and segmental blood vessels such lesions are difficult to compare with blunt traumatic lesions, in which the latter blood vessels are mostly unaffected. The assessment of efficiency of FG used in the treatment of second-degree transcapsular spleen lesions, compared to the traditional suture method, is based on the evaluation of the efficiency of primary haemostasis, appearance of secondary or recurrent bleeding, sufficiency of adhesive-suture line, tissue vitality, process of spleen healing, mortality and other complications.

The aim of this procedure was to assess the safety and efficiency of glue application in the treatment of lienal seconddegree lesions when compared with the traditional method of suture treatment.

\section{Material and methods}

Experimental evaluation of surgical preservation of injured spleen is performed at the Department of Experimental Surgery, Medical Faculty of Nis; Clinic of Pathology, Clinical Centre Nis and Department of Pathology, Military Hospital in Nis. The study was approved by the Ethics Committee of Medical Faculty of Nis.

A total number of 40 healthy mixed breed adult dogs weighing $12-16 \mathrm{~kg}$ were involved in surgical procedures. Anaesthesia was performed with 5\% ketamine hydrochloride (KetalarR) (0.1-0.16 $\mathrm{ml} / \mathrm{kg}$ ). Medial laparotomy and exteriorization of spleen was carried out on all animals. Traumatic spleen rupture of second degree was simulated by one to two $5-\mathrm{cm}$ long and 2-cm deep transcapsular incisions (Splenic Organ Injury Scale).

The vascular stalk was clamped with simultaneous digital compression of the wound edge to minimize the bleeding in the area that is to be filled with FB by means of Duploject application system. FG was applied directly on the wound with the latter application system. The used bi-component FG was prepared at the Institute of Blood Transfusion in Nis. The first component was blood cryoprecipitate (40-50 g/l fibrinogen, factor XIII and fibronectin) and antifibrinolitic (aprotinin). The second component was $\mathrm{CaCl}_{2}$ (40 mmol/l) and bovine thrombin (TrombostatR) (500 $\mathrm{NIH}$ Units/ml). After simultaneous ejection of both components, a fine adhesive layer was made over the spleen wound. Digital compression was held until achieving the sintering of the fibrinous »filling «, and its adhesion to spleen parenchyma. Adhesiveness of FG was checked during spleen reposition in the abdomen. Should the adhesion of FG with spleen parenchyma and its elasticity be satisfactory, and at the same time should the filling of wound without haemorrhage be stable during reposition, the treatment with FG was considered successful.

The control group consisted of 30 animals in which the injuries (identical features) treated by placing two or three $\mathrm{Z}$ sutures, (chromic catgut 2-0). In cases of incomplete haemostasis, additional reparations were performed by placing one or two additional Z sutures with omentoplasty (Added Z Sutures with Omentoplasty - ASO). Should such additional reparations be inefficient, the further reparation attempts are to be cancelled, stitches removed, lesion areas checked, and splenectomy performed consecutively.
Animals were monitored for 10 weeks postoperatively, during which they are subjected to clinical control, two-stage exploration relaparotomy, morphological and histopathological $(\mathrm{PH})$ review of removed spleen. All operated animals (both groups) were subjected to the first planned exploration after 72 hours, which was aimed at checking the compactness (absence of partial or total dehiscence) of adhesive suture-line assessment and effects of haemostasis. The spleens of animals found to display dehiscence, traces of haemorrhage, focal necrosis or local infection in the area of reparations were then removed and subjected to morphological and PH examination. Dead animals were subjected to autopsy, splenectomy and morphological examination of spleen.

All the remaining animals in the control group were randomly divided into 5 subgroups. Every next day, (days 4 to 8 ) one of the subgroups was explored again and splenectomy was performed. Animals in experimental group (treated with FG), were also randomly divided into 5 subgroups. On days 7, 15, 30, 45 and 70, one of the subgroups was surgically explored and subjected to splenectomy. All explored animals of both groups were maintained until complete recovery, and the removed spleens were examined as for morphology and $\mathrm{PH}$ with special emphasis on the area of reparations.

\section{Results}

\section{Experimental group}

Isolated FG application was effective, i.e. allowed establishing the definitive haemostasis in 38 (95\%) cases (Fig. 1).

Only in two $(5 \%)$ cases, the method of preservation was complemented with fibrin omentoplasty namely because of traces of active bleeding,

Although no blood transfusion was applied in any case, all animals survived the operation and recovered quickly and completely without any local or systemic disorders. During the explorative relaparatomy, performed 72 hours after the primary surgery (Figs $2 \mathrm{~A}$ and B), 39 (97.5\%) animals were found to display impeccable lesion fillings with FG with no trace of dechiscence of adhesive lines, complete efficiency of primary haemostasis estimated with lack of the presence of either coagulated or fresh blood in the

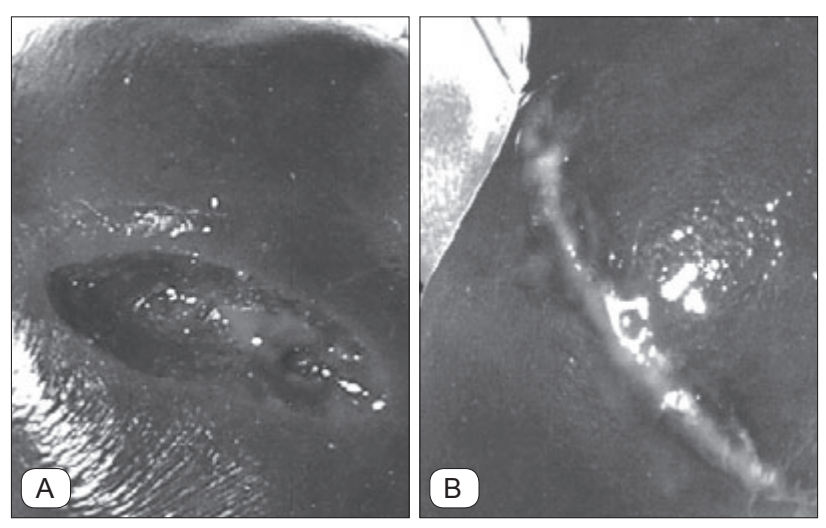

Fig. 1. Appearance of transcapsular lesions of the spleen $\mathrm{II}^{\circ}(\mathrm{A})$. Aspect immediately after the treatment of lesions using the isolated FG (B). 

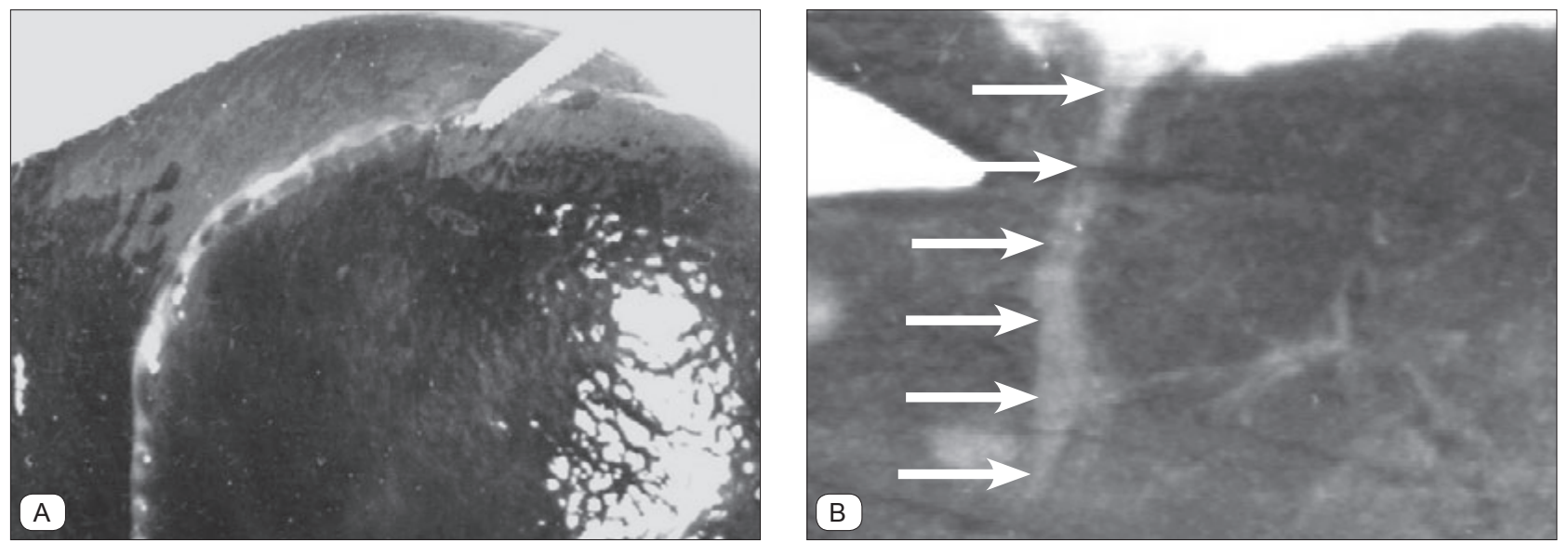

Fig. 2. A) Third postoperative day healing extensive "rupture" of the spleen supervised FG. B) Macroscopical aspect of wound space (formalin preparation), which is completely filled with FG (showed by arrows).

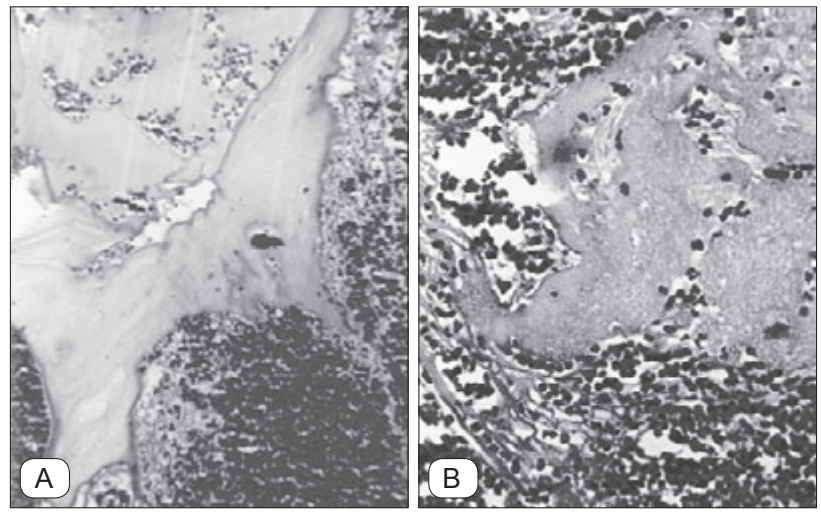

Fig. 3. PH aspect of the spleen lesions treated with FG, after 72 hours. A) Wound space filled with the remains of FG and "marginalized" elements of blood (HEx100). B) Remains FB-A, the elements of blood and fibrin net (HE $\times 200)$. spleen and peritoneal cavity as well as with absence of bleeding from the spleen wound filled with FG, while the vitality of organs was preserved with no evidence of necrosis.

Only in one $(2.5 \%)$ animal, the observation performed after 72 hours revealed a haemodynamically insignificant secondary bleeding from partial dehiscence of the adhesive line. Clinically, the animals did not show any change in behaviour, and after splenectomy, their recovery was safe.

Seventy-two hours after primary surgical intervention, the $\mathrm{PH}$ aspect of many sections made through the entire depth of the wound of the removed spleen showed intimate contact of FB with the edges of the wound. The applied FG was completely filling the entire wound area but at this stage of its evolution we noticed also its initial absorption. In all sections made, granulation tissue progressively invaded the wound space; in this phase of repair it is regularly found in fixed preparations (Figs $3 \mathrm{~A}$ and $\mathrm{B}$ ).

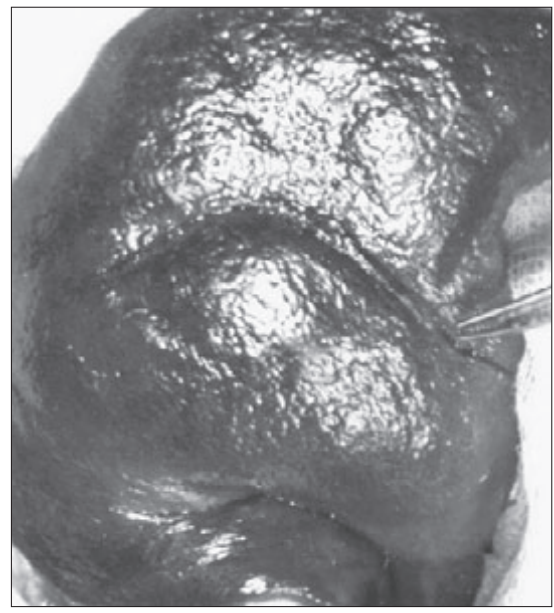

Fig. 4. Seventh postoperative day after treatment FG large lesions of the spleen intraoperative aspect.
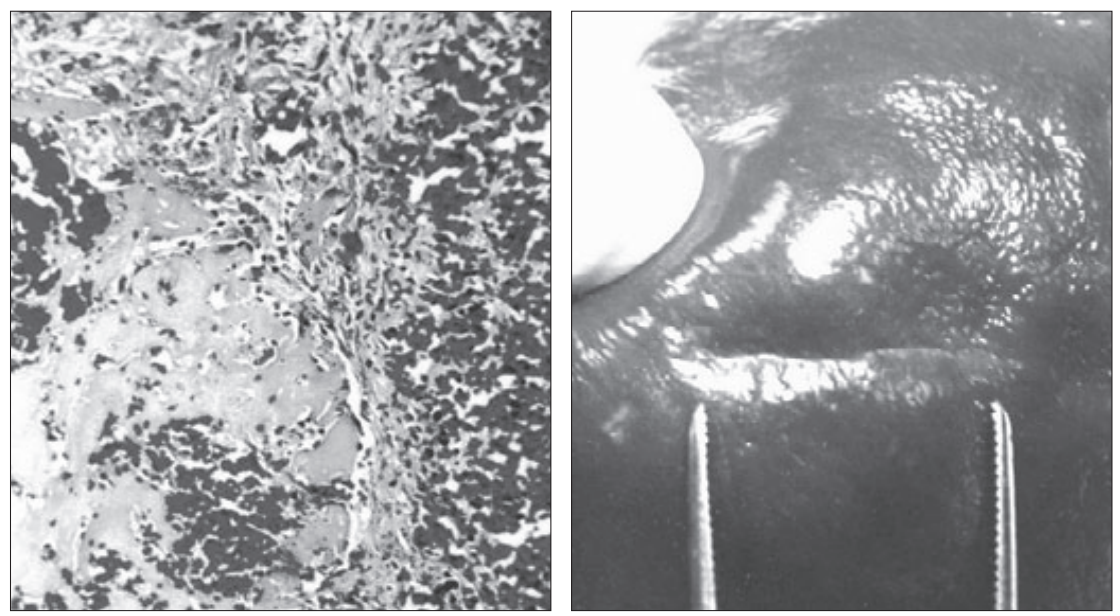

Fig. 5. Granulation tissue, remains of FB and Fig. 6. The appearance of scars after treatment the elements of blood in the wound area. Mar- of FG second degree rupture of the spleen (70 ginal stated fibroblast proliferation. Vital tissue day), intraoperative aspect. of the spleen. (HE x 100). 
109-114

During the planned explorative relaparotomy performed on days $7,15,30,45$ and 70 , the effectiveness of primary haemostasis was confirmed. This was further confirmed by the absence of either fresh or coagulated blood in the peritoneal cavity or spleen lodge. No case displayed evidence of insufficient haemostasis, dehiscence or delayed rupture in the region of adhesive line (Fig. 4).

Gradual absorption of fibrin bung became noticeable no sooner than on day 7 (Fig. 4), when we noticed that the scar was still fresh in phase of gradual consolidation. Nonspecific granulation tissue and blood elements were regularly found in numerous sections, made through the wound. Marginal fibroblast proliferation was clearly stated on preparations made, and the inflammatory response was minimal (Fig. 5).

On day 15, in the absence of traces of fibrin, the bung quite evidently showed mildly depressed scar lines histologically presented with rough bundles of collagen fibres. In the further course of healing, a completely consolidated scar appeared with a noticeably indicated scar retraction in form of a marked reduction in its length and width, and emphasized umbilication. In this and further stages of healing, the $\mathrm{PH}$ images showed a definitive aspect of reparations with a progressive cellular loss and tendency to indicate scar retraction (Fig. 6).

All animals treated with FG resulted in good condition, survived the entire period of monitoring and displayed no complications. The survival rate of these animals was $100 \%$, while the mortality rate was zero.

\section{Control group}

Suture of lineal second-degree lesions with placement of $\mathrm{Z}$ stitches provided successful primary reparation, i.e. complete haemostasis in only $12(40 \%)$ of the total of 30 animals in the control group subdued to this technique. In the remaining (18) animals, additional reparations (ASO) had to be performed because of insufficient haemostasis. The latter reparations were effective only in 7 (23\%) animals. In such cases, we removed the stitch line and disconnected the omental patch with regularly observed deep and spacious cutting of fragile parenchyma and capsule, which resulted in changing the primary lesion into a more extensive and deeper wound, thus producing fourth-degree lesion characteristics. In such case, we discontinued further attempts of suturing, and performed the splenectomy. After the postoperative recovery, the animals subdued to splenectomy were excluded from further follow-up, and 19 dogs were excluded. In relation to the isolated application of FG, which was primarily effective in $38(95 \%)$ cases, the suture, as a primary preservation procedure, showed a significantly lower success rate (40\%) with the necessity of additional reparations in an attempt to control the insufficient haemostasis. In only $2(5 \%)$ cases, insulated FG application required further bonding and great omentum flap coverage. In this group there were cases of unsuccessful preservation. Despite ASO, the preservation was unsuccessful in 11 cases ( $37 \%$ ) from the group of animals treated by suture, while the differences were highly significant (Tab. 1).

Three dogs treated with suture and additional repair died $32-$ 48 hours after surgery. Their autopsy showed heavy bleeding in
Tab. 1. Feasibility repair of lienal injuries second degree level using FG and transcapsular suture.

\begin{tabular}{lccc}
\hline Reparation efficiency & $\begin{array}{c}\text { FG } \\
\text { number } / \%\end{array}$ & $\begin{array}{c}\text { Suture } \\
\text { number } / \%\end{array}$ & $\mathrm{p}$ \\
\hline Efficient primary repair & $38 / 95$ & $12 / 40$ & $0.0000018^{*}$ \\
Efficient additional repair & $2 / 5$ & $7 / 23$ & $0.000002^{*}$ \\
Inability to repair & 0 & $11 / 37$ & $0.0000252^{*}$ \\
\hline
\end{tabular}

Tab. 2. Complications of treatment of the injured spleen using suture and additional repair.

\begin{tabular}{lccc}
\hline Complications & $\begin{array}{c}\text { Sutura } \\
\text { number/\% }\end{array}$ & $\begin{array}{c}\text { Sutura with ASO } \\
\text { number/\% }\end{array}$ & $\mathrm{p}$ \\
\hline Focal necrosis & $5(42 \%)$ & $3(43 \%)$ & 0.35 \\
Secondary hemorrhage & $3(25 \%)$ & $3(43 \%)$ & 0.61 \\
Spleen abscess & $0(0 \%)$ & $2(29 \%)$ & 0.122 \\
Fatal outcome & $1(8 \%)$ & $3(43 \%)$ & 0.12 \\
\hline
\end{tabular}

Tab. 3. Complications of aplied surgical preservation methods in spleen rupture of second degree.

\begin{tabular}{lccc}
\hline Reparation complications & $\begin{array}{c}\mathrm{FG} \\
\text { number/\% }\end{array}$ & $\begin{array}{c}\text { Sutura (ASO) } \\
\text { number } / \%\end{array}$ & $\mathrm{p}$ \\
\hline Focal necrosis & $0 / 0$ & $8 / 42$ & $0.00003^{*}$ \\
Secondary hemorrhage & $1 / 2.5$ & $6 / 32$ & $0.0033^{*}$ \\
Spleen abscess & $0 / 0$ & $2 / 11$ & 0.099 \\
Fatal outcome & $0 / 0$ & $4 / 21$ & $0.0085^{*}$ \\
\hline
\end{tabular}

Tab. 4. Results of treatment of second degree lienal lesions with fibrin glue and suture.

\begin{tabular}{lccc}
\hline Reparation efficiency & $\begin{array}{c}\text { FG } \\
\text { number } / \%\end{array}$ & $\begin{array}{c}\text { Sutura (ASO) } \\
\text { number } / \%\end{array}$ & $\mathrm{p}$ \\
\hline Reparation efficiency & $39 / 97.5$ & $9 / 30$ & $<0.0000001^{*}$ \\
Reparation with complications & $1 / 2.5$ & $10 / 33$ & $0.00058^{*}$ \\
Inability of reparation & 0 & $11 / 37$ & $0.0000252^{*}$ \\
\hline
\end{tabular}

the abdominal cavity and spleen lodge originating from capsule and parenchyma being roughly damaged by sutures. The treated lesion area resembled a site of explosion. Identical findings were recorded on autopsy of the first animal which died 72 hours after the "successfully" derived primary suture repair, with no additional suture and omentoplasty. Secondary haemorrhage with laceration of necrotic parenchyma and capsule is still recorded in 2 animals treated with suture on day 4 , i.e. day 5 during planned explorations. Bleedings that were noted did not have any haemodynamic importance and obvious clinical signs of internal bleeding. After splenectomy, the latter 2 animals recovered. Extensive focal necrosis in the area of placed suture with no signs of secondary bleeding and infection was recorded in 2 animals, treated with suture and scheduled for explorative laparatomy on days 6 and 7 after primary surgery. After splenectomy, the recovery was unremarkable. On days 5 and 7, the subgroup of animals treated with suture and ASO developed purulent formations in size of green walnuts (Tab. 2).

Compared with the subgroup treated by primary suture, the subgroup of animals treated by suture and ASO showed an insignificantly higher incidence of focal necrosis, secondary haemorrhage, localized purulent infections and fatalities.

Looking at the entire group of animals treated with suture, i.e. suture and ASO, the appearance of focal necrosis in $42 \%$ of cases was notable, secondary haemorrhage occurred in $32 \%$, localized 
purulent infection in $11 \%$ and fatal outcome in $21 \%$ of cases. The group of animals treated with FG showed significantly better results in terms of absence of focal necrosis, localized infections, and mortality. The incidence of secondary haemorrhage in the latter group of animals was negligible compared with the group treated with suture (Tab. 3).

The application of FG enabled the spleen rupture of second degree to be repaired in all cases. FG provides a significantly higher rate of successful repair, and minimizes the occurrence of complications in relation to the technique of suture and repeated omentoplasty (Tab. 4).

During the planned exploration, 9 of the remaining animals treated with suture showed no evidence of signs of haemorrhage, laceration, or areas of necrosis of the spleen.

\section{Discussion}

So far, many surgical procedures of the spleen have been described in previous literature (application of haemostatic agents, segmental resection, mesh-splenorrhaphy, stapler resection and auto-transplantation) (14-17).

FG, under various commercial names (Beriplast, Tissucol etc.), is used for repairing parenchymal organs (spleen, liver, pancreas, kidneys and lungs). It has been proven that FG stops the bleeding and fistulisation when applied on cut surface of internal organs, is more effective in drier areas, and that large blood vessels must be sealed. Development of a biological adhesive system opens a great epoch for their application in various fields of surgery of parenchymal organs (18-20).

FG is an optimal alternative to parenchymal suture since the former eliminates many risks of the latter. Unlike other haemostatic agents, such as gelatin sponge, oxidized cellulose and microfibrillar collagen, FG can be effectively used in deep laceration of the spleen without increasing the risk of continuing intrasplenic bleeding, forming subcapsular hematoma or that of delayed rupture of the spleen. In this study, FB was recognized as a supreme procedure of preserving a spleen ruptured as a result of a second-degree trauma, allowing a definite haemostasis and perfect bonding of $97.5 \%$ of transcapsular lesions, and only one $(2.5 \%)$ recorded case with dehiscence of adhesive lines. In our study, the appearance, volume and vitality of preserved organs were fully maintained in all cases treated with FG; no extensive necrosis case was recorded (19-24).

Some studies have emphasized the unacceptably high incidence of unsuccessful primary suture, due to insufficiency of primary haemostasis and extremely damaged capsule and parenchyma of the spleen $(1-3,11)$. Being the simplest way of preservation, the suture of spleen lesions is burdened with the possibility of secondary bleeding due to rupture, i.e. dehiscence of suture line (incidence varies from $0.3 \%$ to over $15 \%$ of cases), and the possibility of necrosis. Deep sutures significantly compress the blood vessels and give conditions for forming a spacious tissue compartment prone to necrosis. Suture of the spleen may be an effective method nevertheless only in combination with other preservation methods such as the use of FG or mesh splenorrhaphy (11).
The treatment of second-degree spleen lesions with suture in this experiment showed significantly worse results compared to those treated with FG. Related to the isolated application of FG, which was primarily effective in $95 \%$, and with fibrin omentoplasty of $100 \%$ of all cases, the suture, as a primary preservation procedure, showed a significantly lower success rate (40\%) with the necessity of additional repair in an attempt to control incomplete haemostasis. When comparing the group treated with FG, where there were no cases of impracticability of primary preservation, to the group of animals treated with suture, the latter, despite additional suture and omentoplasty, yielded $37 \%$ of cases with unsuccessful preservation, while the differences were highly significant. Autopsy findings showed heavy deferred bleeding into abdominal cavity and spleen lodge, in cases of "successfully" derived primary suture repair, and apparently effective haemostasis. This clearly suggests the absence of any safe criterion for assessing the performance, reliability and efficiency of this method. An insignificantly lower incidence of necrosis, secondary haemorrhage, local infection and fatalities in the subgroup treated only with two or three $\mathrm{Z}$ stitches shows that the absence of additional stitches does not eradicate the substantial risk of necrosis in comparison with the technique of additional haemostatic stitches and omentoplasty. Higher mortality in the subgroup treated with suture and additional repair may be explained by higher risk of secondary bleeding from additional stitches in devitalized and lacerated parenchyma. Morphological and $\mathrm{PH}$ evaluation of the healing lesion of the spleen treated by FG indicates that this method allows optimal reintegration, and thus the functionality of this organ. The reaction of tissue to applied FG is successful and the treated parenchyma heals almost with unnoticeable scarring. Any formed scar shows complete compact. FG does not even act as a foreign body, and the vitality of tissues in all cases remains fully preserved. If the surgeon controls the FG application technique, especially with the modalities and details of application, the success rate reaches $90 \%$ in traumatic injuries $(1,2,10,11)$. In this experimental study, the application of FG resulted in an even higher success rate, namely in that of $97.5 \%$.

It turned out that the percentage of primary or forced splenectomy is greatly influenced by surgeon's lack of skills and experience with application of FG. If the surgeon is familiar with this simple and effective method, the rate of saving should exceed $90 \%$ with accidental injury, i.e. $80 \%$, when it comes to traumatic injuries of the spleen $(2,4,16)$. The results of this experimental study suggest a possibility of achieving absolute success, and there is no rational alternative to FG when it comes to treatment of injuries of the spleen of first and second degrees.

The comparative analysis of the applied methods in terms of feasibility of primary repair, efficiency of primary haemostasis, appearance of secondary haemorrhage, mortality rates, sufficiency of adhesive suture-line, assessment of tissue vitality and the process of healing injuries, has undoubtedly shown the superiority of the method of FG application in relation to suture of the spleen and when reviewing the aspects of surgical treatment and preservation of spleen ruptures of first and second degrees. 
$109-114$

\section{References}

1. Hamlat CA, Arbabi S, Koepsell TD, Maier RV, Jurkovich GJ, Rivara FP. National variation in outcomes and costs for splenic injury and the impact of the trauma systems: a population-based cohord study. Ann Surg 2012; 255 (1): 165-170.

2. Clancy TV, Ramshaw DG, Maxwell JG, et al. Management outcomes in splenic injury: a statewide trauma center review. Ann Surg 1997; 226 (1): $17-24$.

3. Stein DM, Scalea TM. Nonoperative management of spleen and liver injuries. J Intensive Care Med 2006; 21( 5): 296-304.

4. Hamour OA, Kashgari RH, Al Harbi MA, Azmi A. Splenic preservation after traumatic rupture. A remote hospital experience. Int Surg 1996; 81 (3): 304-308.

5. Havlicek K. Present possibilities of surgical treatment in splenic injuries Rozhl Chir 1992; 71 (3-4): 185-197.

6. Thompson SR, Holland AJ. Evolution of non-operative management for blunt splenic trauma in children. J Paediatr Child Health 2006; 42 (5): 231-234.

7. Rosito M, Lattarulo S, Pezzolla A, Fabiano G, Palasciano N. The conservative treatment in the splenic trauma. Ann Ital Chir 2009; 80 (3): 231-236.

8. Renzulli P, Gross T, Schnüriger B, et al. Menagement of blunt injuries to the spleen. Br J Surg 2010; 97 (11): 1696-1703.

9. Thompson SR, Holland AJ. Current management of blunt splenic trauma in children. ANZ J Surg 2006; 76 (1-2): 48-52.

10. Kiankhooy A, Sartorelli KH, Vane DW, Bhave AD. Angiographic embolization is safe and effective therapy for blunt abdominal solid organ injury in children. J Trauma 2010; 68 (3): 526-531.

11. Forsythe RM, Harbrecht BG, Peitzman AB. Blunt splenic trauma. Scandinavian J Surg 2006; 95 (3): 146-151.

12. Galvan DA, Peitzman AB. Failure of nonoperative management of abdominal solid organ injuries. Curr Opin Crit Care 2006; (6); 12: 590-594.
13. Bala M, Edden Y, Mintz Y, et al. Blunt splenic trauma: predictors for successful non-operative management. Isr Med Assoc J 2007; 9 (12): $857-861$.

14. McIntyre LK, Schiff M, Jurkovich GJ. Failure of non-operative management of splenic injuries: causes and consequences. Arch surg 2005; 140 (6): 563-568.

15. Kuzma J, Atua V. Conservative management of splenic injury in the tropics. Trop Doc 2008; 38 (4): 210-213.

16. Rajani RR, Claridge JA, Yowler CJ, et al. Improved outcome of adult blunt splenic injury: a cohort analysis. Surgery 2006; 140 (4): 625-631.

17. McCray VW, Davis JW, Lemaster D, Parks SN. Observation for nonoperative management of the spleen: How long is long enough? J Trauma 2008; 65 (6): 1354-1358.

18. Clevert DA, Weckbach S, Minaifar N, Clevert DA, Stickel M, Reiser M. Contrast-enhanced ultrasound versus MS-CT in blunt abdominal trauma. Clin Hemorheol Microcirc 2008; 39 (1-4): 155-169.

19. Valentino M, Ansaloni L, Catena F, Pavlica P, Pinna AD, Barozzi L. Contrast-enhanced ultrasonography in blunt abdominal trauma: considerations after 5 years experience. Radiol Med 2009; 114 (7): 1080-1093.

20. Catalano O, Ajani L, Barozzi L, et al. CEUS in abdominal trauma: multi-center study. Abdom imaging 2009; 34 (2): 225-234.

21. Benjamin W, Mark RH, Sarman A, Paul AT, Wendy L. Angioembolization reduces operative intervention for blunt splenic injury. J Trauma 2008; 64 (6): 1472-1477.

22. Sabe AA, Claridge JA, Rosenblum DI, Lie K, Malangoni MA. The effects of splenic artery embolization on nonoperative management of blunt splenic injury: a 16-year experience. J Trauma 2009; 67 (3): 565-572.

23. Ekeh AP, McCarthy MC, Woods RJ, Haley E. Complications arising from splenic embolization after blunt splenic trauma. Am J Surg 2005; 189 (3): 335-339.

24. Harbrecht BG, Ko SH, Watson GA, Forsythe RM, Rosengart MR, Peitzman AB. Angiography for blunt splenic trauma does not improve the success rate of nonoperative management. J Trauma 2007; 63 (1): 44-49. 